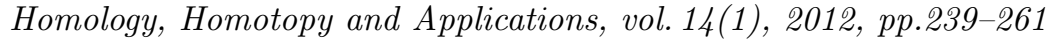

\title{
WEIGHT STRUCTURES AND 'WEIGHTS' ON THE HEARTS OF $t$-STRUCTURES
}

\author{
MIKHAIL V. BONDARKO \\ (communicated by Charles A. Weibel)
}

\begin{abstract}
We define and study transversal weight and $t$-structures (for triangulated categories); if a weight structure is transversal to a $t$-one, then it defines certain 'weights' for its heart. Our results axiomatize and describe in detail the relations between the Chow weight structure $w_{\text {Chow }}$ for Voevodsky's motives (introduced in a preceding paper), the (conjectural) motivic $t$-structure, and the conjectural weight filtration for them. This picture becomes non-conjectural when restricted to the derived categories of Deligne's 1-motives (over a smooth base) and of Artin-Tate motives over number fields. In particular, we prove that the 'weights' for Voevodsky's motives (that are given by $w_{\text {Chow }}$ ) are compatible with those for 1-motives (that were 'classically' defined using a quite distinct method); this result is new. Weight structures transversal to the canonical $t$-structures also exist for the Beilinson's $D_{\tilde{H}_{p}}^{b}$ (the derived category of graded polarizable mixed Hodge complexes) and for the derived category of (Saito's) mixed Hodge modules.

We also study weight filtrations for the heart of $t$ and (the degeneration of) weight spectral sequences. The corresponding relation between $t$ and $w$ is strictly weaker than transversality; yet it is easier to check, and we still obtain a certain filtration for (objects of) the heart of $t$ that is strictly respected by morphisms.

In a succeeding paper we apply the results obtained in order to reduce the existence of Beilinson's mixed motivic sheaves (over a base scheme $S$ ) and 'weights' for them to (certain) standard motivic conjectures over a universal domain $K$.
\end{abstract}

\section{Introduction}

In this paper we study when a weight structure (as defined in [Bon10a]; in [Paw08] weight structures were introduced independently under the name of co-t-structures) yields a certain 'weight filtration' for the heart of a $t$-structure in a triangulated category. We prove several formal results, and describe certain motivic and Hodgetheoretic examples of this situation.

Received December 11, 2011, revised April 6, 2011; published on June 7, 2012. 2000 Mathematics Subject Classification: 4C15, 18E30, 19E15, 14C30, 14 K05.

Key words and phrases: weight structure, motive, triangulated category, $t$-structure. Article available at http://intlpress.com/HHA/v14/n1/a12 and doi:10.4310/HHA.2012.v14.n1.a12 Copyright (C) 2012, International Press. Permission to copy for private use granted. 
The main reason to write this paper was to understand the relation of the 'weight structure approach' to weights for motives (as introduced in [Bon10a]) with the 'classical' one. Recall that the triangulated category $D M_{g m}$ of (geometric) Voevodsky's motives (over a perfect field $k$; all the motives that we will consider in this paper will have rational coefficients) is widely believed to possess a certain motivic $t$ structure $t_{M M}$. Its heart should be the category $M M$ of mixed motives, that should possess a weight filtration whose factors yield certain semi-simple abelian subcategories $M M_{i} \subset M M$ of pure motives of weight $i$. The objects of $M M_{i}$ should be shifts of certain Chow motives (note that in [Doe0] an embedding Chow $\rightarrow D M_{g m}$ was constructed) by $[i]$. Since the existence of $t_{M M}$ is very far from being known, people tried to find a candidate for the weight filtration for $D M_{g m}$; this was to be a filtration by triangulated subcategories that would restrict to the weight filtration for $M M$. This activity was not really successful (in the general case); this is no surprise since (for example) the weight filtration for the motif of a smooth projective variety should correspond to its Chow-Kunneth decomposition.

An alternative method for defining (certain) weights in $D M_{g m}$ was proposed and successfully implemented in [Bon 10a]. To this end weight structures were defined. This notion is a natural important counterpart of $t$-structures; somewhat similar to a $t$-structure, a weight structure $w$ for a triangulated $\underline{C}$ is defined via certain $\underline{C}_{w \leqslant 0}, \underline{C}_{w \geqslant 0} \subset O b j \underline{C}$. The Chow weight structure $w_{C h o w}$ (defined in $\S 6$ of ibid.) certainly does not yield a weight filtration for $D M_{g m}$ in the sense described above (since $D M_{g m, w_{C h o w} \leqslant 0}$ and $D M_{g m, w_{\text {Chow }} \geqslant 0}$ are not stable with respect to shifts). Yet it allows us to define certain (Chow)-weight filtrations and (Chow)-weight spectral sequences for any cohomology of motives; for singular and étale cohomology those are isomorphic to the 'classical' ones (that should also have an expression in terms of the weight filtration for $D M_{g m}$ ), and this should also be true for the "mixed motivic homology' given by $t_{M M}$ (see Remark 2.4 .3 of ibid. and $\delta$ below). Also note here: the Chow weight structure for Voevodsky's motives over a base scheme $S$ (introduced

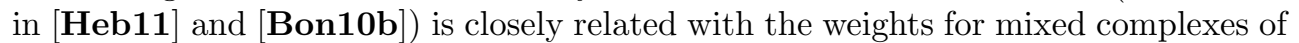
sheaves introduced in $\S 5.1 .8$ of [BBD82] (see $\S \S 3.4-3.6$ of [Bon10b] for more detail), and with weights of mixed Hodge complexes (see $\delta$ below; we prove a very precise statement of this sort in the case when $S$ is the spectrum of a field $k \subset \mathbb{C}$ ).

In the current paper we axiomatize and describe in detail the (conjectural) relations between $w_{C h o w}, t_{M M}$, and the weight filtration for $D M_{g m}$ (we consider the latter in Remark [.4. Delow). To this end we introduce the notion of transversal weight and $t$-structures. It is no surprise that this notion has several non-conjectural (and important) examples; this includes the derived categories of Deligne's 1-motives (over a smooth base) and of Artin-Tate motives over number fields, the derived category of (Saito's) mixed Hodge modules, and the Beilinson's derived category of graded polarizable mixed Hodge complexes. Certain results of [B-VK10] were very useful for studying these examples.

We prove several equivalent conditions for the existence of transversal weight and $t$-structures for a triangulated $\underline{C}$. One of them is the existence of a strongly semiorthogonal generating system of semi-simple abelian subcategories $\underline{A}_{i} \subset \underline{C}$ ( $\underline{A}_{i}$ are the factors of the 'weight filtration' of the heart of $t$ ). We prove that any object of $\underline{H w}$ (the heart of $w$ ) splits into a sum of objects of $\underline{A}_{i}[-i]$ (this should be a generalization of the Chow-Kunneth decomposition of motives of smooth projective varieties). This 
is a strong restriction on $w$; it demonstrates that the notion of transversal structures is quite distinct from the notion of adjacent weight and $t$-structures (introduced in $\S 4.4$ of [Bon]0a]).

We also recall the notion of weight spectral sequences $T(H,-)$ for a (co)homological functor $H$, and prove: if those degenerate at $E_{2}$, then they induce a filtration for $H(-)$ that is strictly respected by morphisms (coming from $\underline{C}$ ). In particular, if (all) $T(H,-)$ degenerate for $H$ being the (zeroth) homology functor $\underline{C} \rightarrow \underline{H t}$, then we obtain a certain weight filtration for $\underline{H t}$. The degeneration of $T$ in this case is strictly weaker than the transversality (of $w$ and $t$ ) and the author does not have a complete understanding of this condition. Its advantage is that it could be 'checked at $t$-exact conservative realizations' of $\underline{C}$. Conjecturally, this statement can be applied to (relative) Voevodsky's motives and their étale realization.

In the subsequent paper [BonIl]] the notion of transversal structures allows us to apply a certain 'gluing' argument, that reduces the existence of a 'nice' motivic $t$-structure for Beilinson motives over (equi-characteristic) 'reasonable' base schemes (cf. [Bon10b] and [CiD09]) to the case of motives over algebraically closed fields. The argument mentioned also relies on the degeneration of (Chow)-weight spectral sequences for 'perverse étale homology' (which conjecturally implies the corresponding degeneration for $H_{0, t_{M M}}$ ).

Most of the results of the current paper are somewhat formal (since we obtain very much new information on the examples described in the paper); they also do not seem to be really unexpected. Yet this paper is definitely the first one where the weights for the heart of a $t$-structure were related with weight structures and weight spectral sequences; this makes it quite important (at least) for the study of various triangulated categories of motives (since one has certain Chow weight structures for them) and of their realizations. In particular, we prove that the 'weights' for Voevodsky's motives (as introduced in [Bمn10a|) are compatible with those for 1motives (that were 'classically' defined using a quite distinct method); this result is rather new (and important). Besides, it seems that our main setting (of transversal weight and $t$-structures) has not been axiomatized previously.

Now we list the contents of the paper.

In the first section we prove the equivalence of nine definitions of transversal weight and $t$-structures. This yields several relations between $t$-structures, weight structures, weight filtrations, and semi-orthogonal generators $\underline{A}_{i}$ in this setting. We don't recall much of the (general) theory of weight structures in this paper; so a reader that is not acquainted with it should probably consult [Bon10a] or [Bon09s] (yet paying attention to the fact that in the definition of a weight structure that we give in the Notation section below and use throughout the paper the 'signs of weights' are opposite to the ones in the papers mentioned). In the end of the section we also calculate the $K_{0}$-group of a triangulated category endowed with transversal weight and $t$-structures.

We start the second section by noting that the results of [B-VKI0] yield a general criterion for the existence of a weight structure that is transversal to the canonical $t$-structure for $D^{b}(\underline{A})$ if $\underline{A}$ admits a 'weight filtration' (with semi-simple 'factors'). We use this result for the construction of the main examples (of transversal weight and $t$-structures) in this paper; yet cf. Remark 2.$](1)$. Applying some more results of $[\mathbb{B}-\nabla K 10]$, we deduce the existence of a weight structure that is transversal to the 
canonical (i.e., 'motivic') $t$-structure for the derived category $D^{b}\left(\mathcal{M}_{1}\right)$ of Deligne's 1-motives. Then we prove that the functors LAlb, RPic (constructed in [B-VKT0]) respect this weight structure (this should also be true for the corresponding motivic $t$-structures if one could define $t_{M M}$ for $D M_{g m}^{\text {eff }}$ ). Note that this fact is not automatic, since the usual definition of weights for 1-motives does not mention Chow motives. Next we verify that on the derived category of mixed Hodge modules over a complex variety $X$, and on the Beilinson's derived category $D_{\tilde{H}_{p}}^{b}$ of graded polarizable mixed Hodge complexes (over a base field $k \subset \mathbb{C}$ ) there exist weight structures transversal to the corresponding canonical $t$-structures; the singular realization functor $D M_{g m}(k) \rightarrow D_{\tilde{H}_{p}}^{b}(k)$ respects the corresponding weight structures. Lastly, we describe the conjectural relations between various 'structures' for $D M_{g m}$.

In $\S 3$ we recall weight filtrations and weight spectral sequences for homology (that correspond to weight structures). We relate the degeneration of weight spectral sequences (at $E_{2}$ ) with the 'exactness' of the corresponding weight filtration. In the case when the homology is the $t$-one, we obtain a certain weight filtration for $\underline{H t}$ (though this degeneration condition is strictly weaker than the transversality of $t$ and $w)$.

The author is deeply grateful to prof. L. Barbieri-Viale who inspired him to write this paper, and to prof. L. Positselski for interesting comments.

Notation. $\underline{C}$ below will always denote some triangulated category. $t$ will denote a bounded $t$-structure for $\underline{C}$.

In the current paper we use the 'homological convention' for weight structures; it was previously used in [Heb1], [Wil08], and in [Bonlla] (whereas the 'signs of

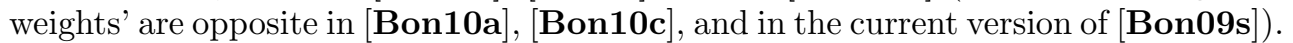
We will say that a pair $\underline{C}_{w \leqslant 0}, \underline{C}_{w \geqslant 0} \subset \operatorname{Obj} \underline{C}$ define a weight structure $w$ if: $\underline{C}_{w \leqslant 0}$, $\underline{C}_{w \geqslant 0}$ are additive and contain all $\underline{C}$-retracts of their objects; $\underline{C}_{w \leqslant 0} \subset \underline{C}_{w \leqslant 0}[1]$, $\underline{C}_{w \geqslant 0}[1] \subset \underline{C}_{w \geqslant 0} ; \underline{C}_{w \leqslant 0} \perp \underline{C}_{w \geqslant 0}[1] ;$ and for any $X \in O b j \underline{C}$ there exists a distinguished triangle $B \rightarrow X \rightarrow A \stackrel{f}{\rightarrow} B[1]$ such that $A \in \underline{C}_{w \geqslant 0}[1], B \in \underline{C}_{w \leqslant 0}$ (that could be called a weight decomposition of $X$; it is not canonically determined by $X$ ).

Below we will need $\underline{C}_{w=0}=\underline{C}_{w \leqslant 0} \cap \underline{C}_{w \geqslant 0}$ (the corresponding additive category $\underline{H w} \subset \underline{C}$ will be called the heart of $w), \underline{C}_{w \leqslant i}=\underline{C}_{w \leqslant 0}[i], \underline{C}_{w \geqslant i}=\underline{C}_{w \geqslant 0}[i]$, and $\underline{C}_{w=i}=$ $\underline{C}_{w=0}[i]$. We will always assume that $w$ is bounded i.e., that $\cup_{i \in \mathbb{Z}} \underline{C}_{w \leqslant i}=\overline{O b j} \underline{C}=$ $\cup_{i \in \mathbb{Z}} \underline{C}_{w \geqslant i}$.

For $X \in \operatorname{Obj} \underline{C}, i \in \mathbb{Z}$, we will consider the following distinguished triangles:

$$
\tau_{\leqslant i} X \rightarrow X \rightarrow \tau_{\geqslant i+1} X
$$

and

$$
w_{\leqslant i} X \rightarrow X \rightarrow w_{\geqslant i+1} X
$$

that come from the $t$-decompositions of $X[i]$ shifted by $[-i]$ (resp. from a weight decomposition of $X[-i]$ shifted by $[i])$, i.e., $\tau_{\leqslant i} X \in \underline{C}^{t \leqslant i}, \tau_{\geqslant i+1} X \in \underline{C}^{t \geqslant i+1}, w_{\leqslant i} X \in$ $\underline{C}_{w \leqslant i}, w_{\geqslant i+1} X \in \underline{C}_{w \geqslant i+1}$ (cf. Remark 1.2.2 of [Bon10a]).

$X^{\tau=i} \in \underline{C}^{t=0}$ will denote the $i$-th homology of $X$ with respect to $t$ i.e., the cone of the corresponding morphism $\tau_{\leqslant-1}(X[i]) \rightarrow \tau_{\leqslant 0}(X[i]) ; \tau_{=i} X=X^{\tau=i}[-i] ; \underline{H t}$ will denote the heart of $t$. 
$D \subset O b j C$ will be called extension-stable if for any distinguished triangle $A \rightarrow B \rightarrow$ $C$ in $\underline{C}$ we have: $A, C \in D \Longrightarrow B \in D$. Note that $\underline{C}^{t \leqslant i}, \underline{C}^{t \geqslant i}, \underline{C}^{t=i}=\underline{C}^{t \geqslant i} \cap \underline{C}^{t \leqslant i}$, $\underline{C}_{w \geqslant i}, \underline{C}_{w \leqslant i}, \underline{C}_{[i, j]}=\underline{C}_{w \geqslant i} \cap \underline{C}_{w \leqslant j}$, and $\underline{C}_{w=i}=\underline{C}_{[i, i]}$ are extension-stable for any $t, w$ and any $i \leqslant j \in \mathbb{Z}$.

For a subcategory $H \subset \underline{C}$ we will call the smallest extension-stable subcategory of $\underline{C}$ containing $H$ the envelope of $H$ (in $\underline{C}$ ).

For $D, E \subset O b j \underline{C}$ we will write $D \perp E$ if $\underline{C}(X, Y)=\{0\}$ for all $X \in D, Y \in E$.

For $B \subset \underline{C}$ we will call the subcategory of $\underline{C}$ whose objects are all retracts of objects of $B$ (in $\underline{C}$ ) the Karoubi-closure of $B$ in $\underline{C}$.

For a class of objects $C_{i} \in \operatorname{Obj} \underline{C}, i \in I$, we will denote by $\left\langle C_{i}\right\rangle$ the smallest strictly full triangulated subcategory containing all $C_{i}$; for $D \subset \underline{C}$ we will write $\langle D\rangle$ instead of $\langle C: C \in O b j D\rangle$.

$\underline{A}$ will always be an abelian category; $\underline{A}_{i}$ (for $i$ running through all integral numbers) will always be additive, and will often be abelian semi-simple.

$k$ will be our perfect base field (sometimes it will be contained in or equal to the field of complex numbers).

\section{Transversal weight and $t$-structures: the general case}

In $\S \mathbb{\square}$ we prove several auxiliary statements; most of them are not really original (yet the definition of semi-orthogonal $\underline{A}_{i}$ is new). We introduce our main notion of transversal weight and $t$-structures, and study it in $\oint$ ए.2.

\subsection{Auxiliary statements}

We will need the following easy homological algebra statements.

Lemma 1.1.1. 1. Let $T: X \stackrel{a}{\rightarrow} A \stackrel{f}{\rightarrow} B \stackrel{b}{\rightarrow} X[1]$ and $T^{\prime}: X^{\prime} \stackrel{a^{\prime}}{\rightarrow} A^{\prime} \stackrel{f^{\prime}}{\rightarrow} B^{\prime} \stackrel{b^{\prime}}{\rightarrow} X^{\prime}[1]$ be distinguished triangles.

Let $B \perp A^{\prime}[1]$. Then for any morphism $g: X \rightarrow X^{\prime}$ there exist $h: A \rightarrow A^{\prime}$ and $i: B \rightarrow B^{\prime}$ completing $g$ to a morphism of triangles $T \rightarrow T^{\prime}$.

Moreover, if $B \perp A^{\prime}$, then $g$ and $h$ are unique.

2. In particular, for any $i \in \mathbb{Z}, X, Y \in O b j \underline{C}$, any $g \in \underline{C}\left(X, X^{\prime}\right)$ can be completed to a morphism of distinguished triangles

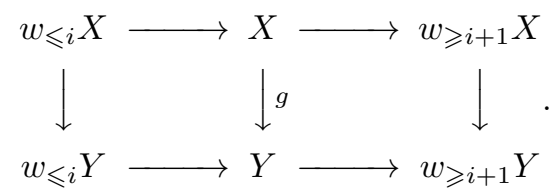

3. If $D \perp E(D, E \subset O b j \underline{C})$, then the same is true for their envelopes.

4. Let $D, E \subset O b j \underline{C}$ be extension-stable, $D \perp(E \cup E[1])$. For some $F \subset O b j \underline{C}$ suppose that for any $X \in F$ there exists a distinguished triangle $Y \rightarrow X \rightarrow Z$ with $Y \in D, Z \in E$. Then such a distinguished triangle also exists for any $X$ belonging to the envelope of $F$ (in $\underline{C}$ ).

5. For any $i \leqslant j \in \mathbb{Z}$ we have: $\underline{C}_{[i, j]}$ is the envelope of $\cup_{i \leqslant l \leqslant j} \underline{C}_{w=l}$ in $\underline{C}$.

6. Let $B$ be an additive category; let $B_{i} \subset B, i \in \mathbb{Z}$, be its full additive subcategories such that $B_{i} \perp B_{j}$ for $j>i$, and $\operatorname{Obj} B=\bigoplus_{i \in \mathbb{Z}} \operatorname{Obj} B_{i}$. Suppose that all $B_{i}$ are idempotent complete (i.e., that for any $X \in O b j B_{i}$ and any idempotent $s \in B_{i}(X, X)$ 
there exists a decomposition $X=Y \oplus Z$ such that $s=j \circ p$, where $j$ is the inclusion $Y \rightarrow X(\cong Y \bigoplus Z), p$ is the projection $X(\cong Y \bigoplus Z) \rightarrow Y)$. Then $B$ is idempotent complete also.

Proof. 1. This is Lemma 1.4.1 of [Bon10a]; it follows immediately from Proposition 1.1.9 of [BBD82].

2. Follows immediately from assertion 1; cf. Lemma 1.5.1 of [Bon10a].

3. Very easy; note that for any $X \in \operatorname{Obj} \underline{C}$ the (corepresentable) functor $\underline{C}(X,-)$ is homological, whereas $\underline{C}(-, X)$ is cohomological.

4. See Remark 1.5.5 of [Bon10a] or Proposition 1.8 of [सeb]1].

5. Easy from Proposition 1.5.6(2) of [Bon10a].

6. We prove the assertion in question for $X=\bigoplus_{i_{1} \leqslant i \leqslant i_{2}} X_{i}$, where $X_{i} \in \operatorname{Obj} B_{i}$, by induction in $i_{2}-i_{1}$. For $i_{2}-i_{1} \leqslant 1$ the statement follows from our assumptions.

Suppose now that our claim holds if $i_{2}-i_{1}<m$ for some $m>1$. Let $i_{2}-i_{1}=m$. We decompose $s$ as $\bigoplus s_{i j}, s_{i j} \in B\left(s_{i}, s_{j}\right)$. Our orthogonality assumption yields that the morphism $s_{1}=s_{i_{2}, i_{2}}: X_{i_{2}} \rightarrow X_{i_{2}}$ is idempotent, as well as $s_{2}=\bigoplus_{i_{1} \leqslant i, j<i_{2}} s_{i j}$. The inductive assumption yields that $s_{1}$ and $s_{2}$ correspond to certain $X_{1}, Y_{1}, X_{2}, Y_{2} \in$ $O b j B$ respectively.

Now, it suffices to verify that the morphism $s$ is conjugate to $s_{1}+s_{2}$. Denote $s-s_{1}-s_{2}$ by $d$. Then our orthogonality assumptions yield: $s_{1} \circ s_{2}=s_{2} \circ s_{1}=$ $d \circ s_{2}=s_{1} \circ d=d^{2}=0$. Besides, $d=d \circ s_{1}+s_{2} \circ d$; composing with $s_{2}$ we obtain that $s_{2} \circ d \circ s_{1}=0$. Hence $\left(i d_{X}-d \circ s_{1}+s_{2} \circ d\right)\left(i d_{X}+d \circ s_{1}-s_{2} \circ d\right)=i d_{X}$; therefore for $h=i d_{X}-d \circ s_{1}+s_{2} \circ d$ we have $h \circ\left(s_{1}+s_{2}\right) \circ h^{-1}=s$.

Below we will need a certain class of 'nice' weight decompositions.

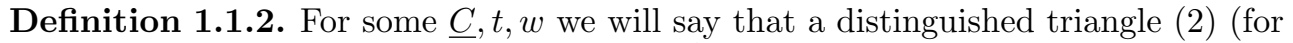
some $i, X)$ is nice if $w_{\leqslant i} X, X, w_{\geqslant i+1} X \in \underline{C}^{t=0}$.

We will also say that this distinguished triangle is a nice decomposition of $X$ (for the corresponding $i$ ), and that the morphism $w_{\leqslant i} X \rightarrow X$ extends to a nice decomposition.

Now we formulate a simple implication of Lemma case of it below, and a somewhat more complicated one in [BonItb]).

Lemma 1.1.3. We fix some $\underline{C}, w, t, i$; suppose that for a certain $N \subset \underline{C}^{t=0}$ a nice decomposition exists for any $X \in N$. Consider $N^{\prime} \subset \underline{C}^{t=0}$ being the smallest subclass containing $N$ that satisfies the following condition: if $A, C \in N^{\prime}$,

$$
A \stackrel{f}{\rightarrow} B \stackrel{g}{\rightarrow} C
$$

is a complex (i.e., $g \circ f=0$ ), $f$ is monomorphic, $g$ is epimorphic, $\operatorname{Ker} g / \operatorname{Im} f \in N^{\prime}$, then $B \in N^{\prime}$. Then a nice decomposition exists for any $X \in N^{\prime}$ (and the same $i$ ).

Proof. It suffices to note that $N^{\prime}$ is exactly the smallest extension-stable subcategory of $\underline{C}$ containing $N$, and apply Lemma ㅁ.

Next we study certain ('weight') filtrations of triangulated categories.

Definition 1.1.4. 1 . We will say that a family $\left\{\underline{A}_{i}\right\}, \underline{A}_{i} \subset \underline{C}, i \in \mathbb{Z}$, is semi-orthogonal if $\underline{A}_{i} \perp \underline{A}_{j}[s]$ for any $i, j, s \in \mathbb{Z}$ such that $s<0$, or $s>i-j$. 
We will say that $\left\{\underline{A}_{i}\right\}$ are strongly semi-orthogonal if we also have $\underline{A}_{i} \perp \underline{A}_{j}$ for any $i>j$ (and so, for any $i \neq j$ ).

2. We will say that $\left\{\underline{A}_{i}\right\}$ is generating (in $\underline{C}$ ) if $\left\langle\cup_{i} \operatorname{Obj} A_{i}\right\rangle=\underline{C}$.

Now we prove that a semi-orthogonal generating family yields a certain ('weight') filtration for $\underline{C}$ in the sense of Definition E17.1 of [B-VKT0], and study its properties.

Lemma 1.1.5. Let $i>j \geqslant n \in \mathbb{Z}$.

I Suppose that $\left\{\underline{A}_{s} \subset \underline{C}\right\}$ is a semi-orthogonal family; denote $\left\langle\underline{A}_{s}\right\rangle$ by $\underline{C}_{s}$ (for any $s \in \mathbb{Z})$.

Then $\underline{C}_{j} \perp \underline{C}_{i}$.

II Let $\underline{C}_{l} \subset \underline{C}$ for $l \in \mathbb{Z}$ be triangulated; suppose that $\underline{C}_{l} \perp \underline{C}_{m}$ if $l<m$. $\underline{C}_{\leqslant r}$.

For any $r \leqslant q \in \mathbb{Z}$ denote $\left\langle\cup_{r \leqslant s \leqslant q} \operatorname{Obj} \underline{C}_{s}\right\rangle$ by $\underline{C}_{[r, q]}$, and denote $\left\langle\cup_{s \leqslant r} \operatorname{Obj} \underline{C}_{s}\right\rangle$ by

Then the following statements are fulfilled.

1. For any $X \in \operatorname{Obj}_{[n, i]}$ there exists a distinguished triangle

$$
X_{1} \rightarrow X \rightarrow X_{2}
$$

such that $X_{1} \in O \operatorname{Obj} \underline{C}_{[n, j]}, X_{2} \in O b j \underline{C}_{[j+1, i]}$. More generally, for $X \in \underline{C}_{\leqslant i}$ one can find (因) with $X_{1} \in O b j \underline{C}_{\leqslant j}$.

Besides, this triangle is (canonical and) functorial in $X$ (in both cases).

2. The embedding $\underline{C}_{[j+1, i]} \rightarrow \underline{C}_{\leqslant i}$ possesses an exact left adjoint $a_{i, j}$; the 'kernel' of $a_{i, j}$ is exactly $\underline{C}_{\leqslant j}$.

3. Suppose that $\left\{\underline{C}_{l}\right\}$ are generating. Then the embedding $\underline{C}_{\leqslant i} \rightarrow \underline{C}$ possesses an exact right adjoint $b_{i}$.

Proof. I If $i<l$, then $\underline{A}_{i}[r] \perp \underline{A}_{l}$ for any $r \in \mathbb{Z}$ (by Definition $\left.\square.\right]$ ). Now the result is immediate from Lemma

II 1. Since $w$ is bounded, we have $\underline{C}_{\leqslant i}=\cup_{m \leqslant i} \underline{C}_{[m, i]}$; hence it suffices to verify the existence of (甘) for $X \in \underline{C}_{[n, i]}$.

We have a 'trivial' example of (四) if $X \in \underline{C}_{l}$ for any $i \geqslant l \geqslant n$. Hence the existence of

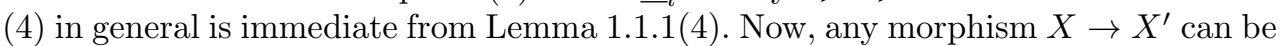
uniquely extended to a morphism of the corresponding triangles by Lemma Hence we obtain the functoriality of (四).

2,3: Immediate from assertion II1 by well-known homological algebra statements; see Proposition E.15.1 of [B-VK10] .

\subsection{Transversal weight and $t$-structures: equivalent definitions and their consequences}

Theorem 1.2.1. The following conditions are equivalent.

(i) There exists a strongly semi-orthogonal generating family $\left\{\underline{A}_{i}\right\}$ in $\underline{C}$ such that all of $\underline{A}_{i}$ are abelian semi-simple.

(ii) There exists a semi-orthogonal family $\left\{\underline{A}_{i}\right\}$ in $\underline{C}$ such that for $\underline{C}^{t \leqslant 0}$ (resp. $\underline{C}^{t \geqslant 0}$ ) being the envelope of $\cup_{i \in \mathbb{Z}, j \geqslant 0} \underline{A}_{i}[j]$ (resp. of $\left.\cup_{i \in \mathbb{Z}, j \leqslant 0} \underline{A}_{i}[j]\right)$ we have: $\left(\underline{C}^{t \leqslant 0}\right.$, $\underline{C}^{t \geqslant 0}$ ) yield a $t$-structure for $\underline{C}$. 
(ii') There exists a semi-orthogonal family $\left\{\underline{A}_{i}\right\}$ in $\underline{C}$ such that the envelope of $\cup_{i \in \mathbb{Z}} \underline{A}_{i}$ yields the heart of a certain $t$.

(iii) There exist a $t$ and a semi-orthogonal family $\left\{\underline{A}_{i} \subset \underline{H t}\right\}$ that satisfy the following condition: for each $X \in \underline{C}^{t=0}$ there exists an exhaustive separated increasing filtration by subobjects $W_{\leqslant i} X, i \in \mathbb{Z}$, such that $W_{\leqslant i} X / W_{\leqslant i-1} X$ belongs to Obj$\underline{A}_{i}$ for all $i \in \mathbb{Z}$.

(iii') The filtration (of any $X \in \underline{C}^{t=0}$ ) described above exists and is $\underline{H t}$-functorially determined by $X$.

(iv) There are $t, w$ for $\underline{C}$ such that for any $X \in \underline{C}^{t=0}, i \in \mathbb{Z}$, there exists a nice decomposition (see Definition [1.J.).

(iv') Nice decompositions exist, and they are also $\underline{H t}$-functorial in $X$ (if we fix $i$ ); the corresponding functors $X \mapsto w_{\leqslant i} X$ and $X \mapsto w_{\geqslant i+1} X$ are exact (on $\underline{H t}$ ).

(v) There are $t, w$ for $\underline{C}$ such that for any $X \in \underline{C}^{t=0}, i \in \mathbb{Z}$, there exists a choice of $w_{\leqslant i} X$ such that the morphism $\operatorname{Im}\left(\left(w_{\leqslant i} X\right)^{\tau=0} \rightarrow X\right) \rightarrow X$ extends to a nice decomposition of $X$.

$\left(v^{\prime}\right)$ For $t, w$ and any $X, i, w_{\leqslant i} X$ (as above) the morphism $\operatorname{Im}\left(\left(w_{\leqslant i} X\right)^{\tau=0} \rightarrow X\right) \rightarrow$ $X$ extends to a nice decomposition of $X$.

Proof. Certainly, (ii') implies (ii) (since $t$ is bounded), (iii') implies (iii), (iv') implies (iv), and (v') implies (v).

(i) $\Longrightarrow$ (ii).

Semi-orthogonality yields that the 'generators' of $\underline{C}^{t \leqslant 0}[1]$ are orthogonal to those of $\underline{C}^{t \geqslant 0}$; hence Lemma प्टा(3) yields: $\underline{C}^{t \leqslant 0}[1] \perp \underline{C}^{t \geqslant 0}$.

It remains to verify the existence of $t$-decompositions.

For any $i \in \mathbb{Z}$ we have $\underline{C}_{i} \cong \bigoplus_{j \in \mathbb{Z}} \underline{A}_{i}[j]$ (in the notation of loc.cit.). Indeed, the obvious comparison functor $\bigoplus_{j \in \mathbb{Z}} \underline{A}_{i}[j] \rightarrow \underline{C}_{i}$ is an equivalence of triangulated categories, since $\underline{A}_{i}$ is semi-simple and $\underline{A}_{i} \perp \underline{A}_{i}[j]$ for any $j \neq 0$.

Hence any object of $\underline{C}_{i}$ admits a $t$-decomposition $X \cong \tau_{\leqslant 0} X \bigoplus \tau_{\geqslant 1} X$ whose components also belong to $\underline{C}_{i}$.

Now, it suffices to verify: if for some $j<i$ any object of $\underline{C}_{[j+1, i]}$ admits a $t$ decomposition inside $\underline{C}_{[j+1, i]}$, then a similar statement holds for any $X \in O b j \underline{C}_{[j, i]}$.

Lemma प. (III) yields the existence of a distinguished triangle $X_{1} \rightarrow X \rightarrow X_{2} \stackrel{g}{\rightarrow}$ $X_{1}[1]$ such that $X_{1} \in O \operatorname{Obj} \underline{C}_{j}, X_{2} \in \operatorname{Obj} \underline{C}_{[j+1, i]}$.

Now we argue as in the proof of Lemma 1.5.4 of [Bon]0a]. We can complete $g$ to a morphism of distinguished triangles

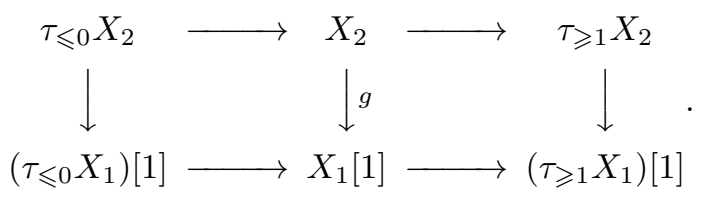

Indeed, by Lemma $\mathbb{\square} \boldsymbol{L}$ (1) it suffices to verify that $\tau_{\leqslant 0} X_{2} \perp\left(\tau_{\geqslant 1} X_{1}\right)[1]$; the last statement easily follows from the strong semi-orthogonality of $\left\{\underline{A}_{s}\right\}$ (see Lemma $\square \cdot \mathbb{C}(3)$ ). 
Moreover, we can complete $g$ to the following diagram (starting from the left hand side square of (国), and using Proposition 1.1.11 of [BBD82]):

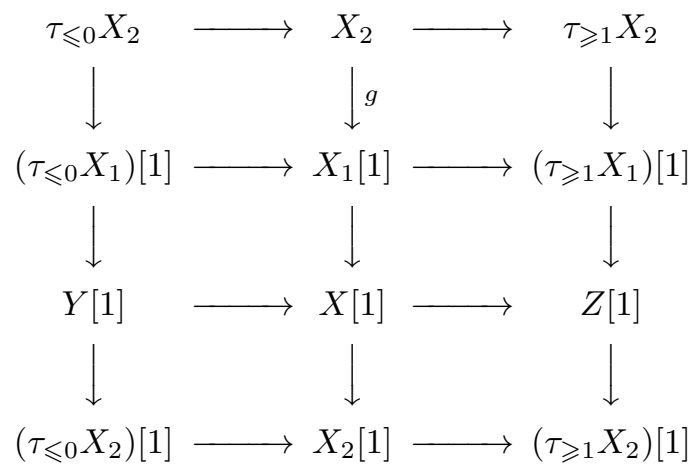

such that all rows and columns are distinguished triangles, and all squares are commutative. Therefore the extension-stability of $C^{t \leqslant 0}$ yields that it contains $Y$; the extension-stability of $\underline{C}^{t \geqslant 1}$ yields that it contains $Z$; hence $Y \rightarrow X \rightarrow Z$ is the $t$ decomposition desired.

(ii) $\Longrightarrow$ (iv).

We take $C_{1}$ being the envelope of $\left\{\underline{A}_{i}[j], i+j \geqslant 0, i, j \in \mathbb{Z}\right\}$ in $\underline{C}, C_{2}$ being the envelope of $\left\{\underline{A}_{i}[j], \quad i+j \leqslant 0\right\}$. Note that $C_{1}$ is the envelope of $\{H[j], j \geqslant 0\}, C_{2}$ is the envelope of $\{H[j], j \leqslant 0\}$, where $\operatorname{Obj} H=\bigoplus_{i \in \mathbb{Z}} \operatorname{Obj} \underline{A}_{i}[-i]$. Besides, $H$ is negative i.e., $H \perp H[j]$ for all $j>0$.

Hence, as shown (in the proof of) Theorem 4.3.2(II) of [Bon10a], there exists a weight structure $w$ (for $\underline{C}$ ) such that $\underline{C}_{w \geqslant 0}$ (resp. $\underline{C}_{w \leqslant 0}$ ) is the Karoubi-closure of $C_{1}$ (resp. $C_{2}$ ) in $\underline{C}$ yield a bounded weight structure $w$ for $\underline{C}$ (actually, this is a simple

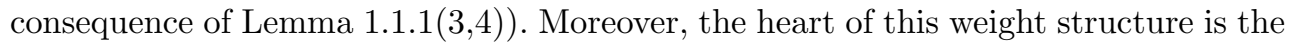
idempotent completion of $H$. Now, $H$ is idempotent complete itself, since all $\underline{A}_{i}$ are (note that $\underline{A}_{i}[-i] \perp \underline{A}_{j}[-j]$ for $j>i$, hence we can apply Lemma ㄷ. $H=\underline{H w}$. Then Lemma ㄸ. $\mathbf{D}(5)$ implies that $\underline{C}_{w \geqslant 0}=C_{1}$ and $\underline{C}_{w \leqslant 0}=C_{2}$ (i.e., we don't need Karoubi-closures here; here we use the fact that $\underline{C}_{w \leqslant 0}=\cup_{i \leqslant 0} \underline{C}_{[i, 0]}$ and $\underline{C}_{w \geqslant 0}=\cup_{i \geqslant 0} \underline{C}_{[0, i]}$ for a bounded $\left.w\right)$. Lastly, by Lemma ㅁ. ${ }^{3}$ it suffices to verify the existence of nice decompositions for those objects of $\underline{H t}$ that belong to one of $\underline{A}_{i}$; this is obvious.

(v) $\Longrightarrow$ (iv): obvious.

(iv) $\Longrightarrow\left(\mathbf{v}^{\prime}\right)$. It suffices to note: by Proposition 2.1.2(1) of [Bon10a], $\operatorname{Im}\left(\left(w_{\leqslant i} X\right)^{\tau=0} \rightarrow X\right)$ does not depend on the choice of $w_{\leqslant i} X$ (cf. Definition $[.]^{-}$ below). Hence it suffices to consider the case when $w_{\leqslant i} X$ comes from a nice decomposition of $X$, and then the statement is obvious.

(iv) $\Longrightarrow$ (iv') and (iii).

We set $\underline{A}_{i}=\underline{C}^{t=0} \cap \underline{C}_{w=i}$. The orthogonality axioms of weight and $t$-structures immediately yield that the family $\left\{\underline{A}_{i}\right\}$ is semi-orthogonal.

Now we prove (iii). Since all terms of a nice decomposition belong to $\underline{C}^{t=0}$, it yields a short exact sequence in $\underline{H t}$. In particular, the corresponding morphism $w_{\leqslant i} X \rightarrow X$ is monomorphic in $\underline{H t}$.

Now suppose that $X \in \underline{C}_{w \leqslant i}$. Then we have $w_{\geqslant i} X \in \underline{C}_{w=i}$; see Proposition 1.3.3(6) of [Bon10a]. Hence, $w_{\geqslant i} X$ belongs to $\underline{A}_{i}$ for any nice (घ). 
Loc. cit. also yields: if $X \in \underline{C}_{w \geqslant j}, j<i$, then any choice of $w_{\leqslant i} X$ belongs to $\underline{C}_{w \geqslant j}$ also. Hence for $X \in \underline{C}_{[r, s]} \cap \underline{C}^{t=0}, r \leqslant s \in \mathbb{Z}$, one can take $W_{\leqslant l} X=X$ for $l \geqslant s$, then by induction starting from $i=s-1$ down to $i=r$ take a choice of $W_{\leqslant i} X$ coming from a nice decomposition of $W_{\leqslant i+1} X$ (that was constructed on the previous step), and set $W_{\leqslant l}=0$ for $l<r$; this filtration would satisfy the conditions of (iii).

Now we verify (iv'). Any morphism in $\underline{C}$ can be extended to a morphism of (any choices of) weight decompositions by Lemma unique in our case by parts 1 and 3 of loc.cit. (here we apply the orthogonality statement proved above). Hence we obtain that nice choices of (지) (for a fixed $i$ ) yield a functor (here we take $X \in \underline{C}^{t=0}$ ).

Now, Lemma 1.5.4 of [Bon]0a] yields that for any distinguished triangle $A \rightarrow$

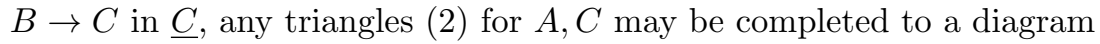

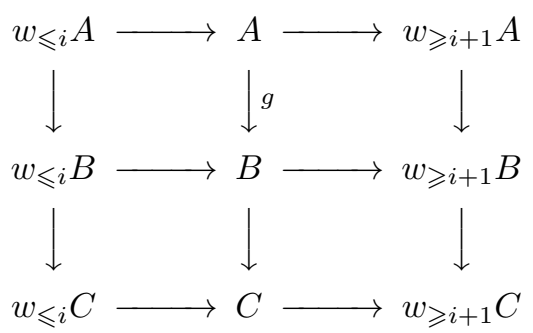

all of whose rows and columns are distinguished triangles (and the middle row is

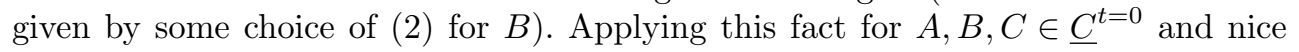
decompositions of $A, C$, we obtain that the middle row is a nice decomposition of $B$ (since $\underline{H t}$ is extension-stable in $\underline{C}$ ). Then the exactness of columns (in $\underline{H t}$ ) concludes the proof of (iv').

Next we note that (ii) along with (iii) implies (ii'). Indeed, the envelope of $\underline{A}_{i}$ obviously lies in $\underline{H t}$, whereas (iii) yields that this inclusion is an equality.

It remains to verify that (iii) implies (iii') and (i).

To this end first we verify that (iii) implies (iv). Obviously, the family $\left\{\underline{A}_{i}\right\}$ is generating (since $t$ is bounded). We consider $C_{1}, C_{2} \subset \underline{C}$ introduced in the proof of (ii) $\Longrightarrow$ (iv). As we have already noted above, the Karoubi-closures of $C_{1}$ and $C_{2}$ in $\underline{C}$ yield a weight structure for $\underline{C}$. Hence the distinguished triangles coming from the short exact sequences $0 \rightarrow W_{\leqslant i} X \rightarrow X \rightarrow X / W_{\leqslant i} X \rightarrow 0$ yield (घ)). We obtain that (iii) implies (iv); hence (iii) also yields (iv').

Obviously, (iv') implies (iii'). Also, (iv') yields that $\underline{A}_{i} \perp \underline{A}_{j}$ for $j>i$ by the Remark E7.8 and Proposition E7.4(4) of [B-VKT0] (cf. Proposition [...3 below).

So, it remains to prove that $\underline{A}_{i}$ are abelian semi-simple. We verify that (for a fixed $i \in \mathbb{Z}$ ) the classes $\operatorname{Obj} \underline{C}_{i} \cap \underline{C}^{t \leqslant 0}$ and $\operatorname{Obj} \underline{C}_{i} \cap \underline{C}^{t \geqslant 0}$ yield a $t$-structure for $\underline{C}_{i}$ (i.e., that $t$ can be restricted to $\underline{C}_{i}$ ). To this end we note that for any $i \geqslant j \in \mathbb{Z}$, $X \in \underline{C}_{\leqslant i}$ (see Lemma ㄸ. . (II)) the distinguished triangle $W_{\leqslant j} X \rightarrow X \rightarrow X / W_{\leqslant j} X$ (as considered above) is simultaneously a choice of (四). It easily follows that all $b_{j}$ and $a_{i, j}$ respect $\underline{H t}$. Hence they also respect $t$-decompositions; see Lemma E19.1

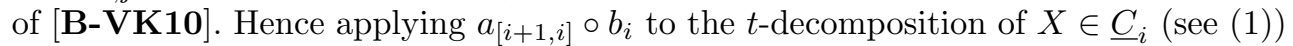
we obtain that its components belong to $\underline{C}_{i}$. We also obtain that the heart of this $t$-structure is $a_{[i, i-1]} \circ b_{i}(\underline{H t})=\underline{A}_{i}$. Hence $\underline{A}_{i}$ is an abelian category, and short exact sequences in it yield distinguished triangles in $\underline{C}$. Therefore $\underline{A}_{i}$ is abelian semi-simple, since $\underline{A}_{i} \perp \underline{A}_{i}[1]$ by semi-orthogonality. 
Definition 1.2.2. If $w, t$ satisfy the (equivalent) conditions of the theorem, we will say that $t$ is transversal to $w$.

Remark 1.2.3. 1. One could (try to) modify the conditions of the Theorem in order to include the case when $w$ and $t$ are not (necessarily) bounded. Yet to this end one would definitely require certain technical restrictions on $\underline{C}$ (cf. Theorems 4.3 .2 and 4.5.3 of [Bon10a] $)$.

2. Some more details on connections between $w, t$, and $\left\{\underline{A}_{i}\right\}$ are contained in the proof of the Theorem. In particular, note that the functor $X \mapsto \bigoplus_{i \in \mathbb{Z}} \tau_{=i} X$ yields a splitting $\underline{C}_{w=0}=\bigoplus O \operatorname{Obj} \underline{A}_{i}[-i]$ (though we don't have an isomorphism of the corresponding categories, since there could be non-zero morphisms from $\underline{A}_{i}[-i]$ into $\underline{A}_{j}[-j]$ for $j<i$ ). Besides, $\underline{H t}$ possesses a separated exhaustive filtration with semi-simple factors $\underline{A}_{i}=\underline{H t} \cap \underline{H w}[i]$.

3. Condition (i) of the Theorem is self-dual. If follows: if $w, t$ are transversal for $\underline{C}$, then the structures $w^{o p}, t^{o p}$ for the opposite category $\underline{C}^{o p}$ are transversal also. The latter structures are defined as follows: $\underline{C}_{w^{o p} \leqslant 0}^{o p}=\underline{C}_{w \geqslant 0}$ and $\underline{C}_{w^{o p} \geqslant 0}^{o p}=\underline{C}_{w \leqslant 0}$; $\underline{C}^{o p, t^{o p} \leqslant 0}=\underline{C}^{t \geqslant 0}$ and $\underline{C}^{o p, t^{o p} \geqslant 0}=\underline{C}^{t \leqslant 0}$ (cf. Remark 1.1.2(1) of [BRon10]] ).

Besides, for any $i, j \in \mathbb{Z}$ the structures $w[i], t[j]$ are also transversal; here $\underline{C}_{w[i] \leqslant 0}=$ $\underline{C}_{w \leqslant i}$ and $\underline{C}_{w[i] \geqslant 0}=\underline{C}_{w \geqslant i} ; \underline{C}^{t[j] \leqslant 0}=\underline{C}^{t \leqslant j}$ and $\underline{C}^{t[j] \geqslant 0}=\underline{C}^{t \geqslant j}$.

4. Proposition 2.1.2(1) of [Bon10a] actually yields (cf. Definition [.] below) that for any $t, w$ the correspondence $X \mapsto \operatorname{Im}\left(\left(w_{\leqslant i} X\right)^{\tau=0} \rightarrow X\right)$ yields a functor in $\underline{H t} \rightarrow \underline{H t}$; hence this is also true for $X \mapsto \operatorname{Coker}\left(\left(w_{\leqslant i} X\right)^{\tau=0} \rightarrow X\right)$. So, in order to verify that $t, w$ are transversal it suffices to verify that these functors take their values in $\underline{C}_{w \leqslant i}$ and $\underline{C}_{w \geqslant i+1}$, respectively (for all $i \in \mathbb{Z}$ ).

Most of the following results were also (essentially) verified in the process of proving Theorem ㄷ.2.

Proposition 1.2.4. Let $t$ be transversal to $w, i \in \mathbb{Z}$. Then the following statements are fulfilled.

I1. The functors $X \mapsto \tau_{\leqslant i} X$ and $X \mapsto \tau_{\geqslant i} X$ map $\underline{C}_{w \leqslant 0}$ and $\underline{C}_{w \geqslant 0}$ into themselves.

2. $X \in \underline{C}_{w \leqslant i}$ (resp. $X \in \underline{C}_{w \geqslant i}$ ) whenever for any $j \in \mathbb{Z}$ we have $W_{\leqslant i+j}\left(X^{\tau=j}\right)=$ $X$ (resp. $W_{\leqslant i+j-1}\left(X^{\tau=j}\right)=X^{\tau=j}$; here $W_{\leqslant i}(-)$ is the filtration given by condition (iii') of Theorem [.2]).

II1. The functor $X \mapsto W_{\leqslant i} X$ (from $\underline{H t}$ into $\underline{H t}$ ) given by condition (iii') of the Theorem, is right adjoint to the embedding $\underline{C}_{w \leqslant i} \underline{\underline{H t}} \rightarrow \underline{H t}$; it is exact.

2. For $X \in \underline{C}^{t=0}$ denote $X / W_{\leqslant i} X$ by $W_{\geqslant i+1} X$. Then the functor $W_{\geqslant i+1}(-)$ is left adjoint to the embedding $\underline{C}_{w \geqslant i+1} \cap \underline{H t} \rightarrow \underline{H t}$; it is exact.

III 1. The functors $G r_{i}: X \mapsto W_{\geqslant i}\left(W_{\leqslant i} X\right)$ and $G r_{i}^{\prime}: X \mapsto W_{\leqslant i}\left(W_{\geqslant i} X\right)$ (defined as the compositions of the functors from assertion II) are canonically isomorphic exact projections of $\underline{H t}$ onto $\underline{A}_{i}$. Moreover, $G r_{i}(X) \cong W_{\leqslant i} X / W_{\leqslant i-1} X$.

2. For $X \in \underline{C}^{t=0}$ we have: $X \in \underline{C}_{w \leqslant i}\left(\right.$ resp. $X \in \underline{C}_{w \geqslant i}$ ) whenever $\operatorname{Gr}_{j}(X)=0$ for all $j>i$ (resp. for all $j<i$ ).

Proof. I1. Since $t$ is bounded, it suffices to verify a similar statement for the functors $\tau=j$ for all $j \in \mathbb{Z}$ (since the functors mentioned in the assertions can be obtained from these functors via 'extensions'). 
Lemma $\square \cdot \boldsymbol{L}$ (5) allows reducing the latter statement to its analogue for $\underline{C}_{w=0}$ (and all $j \in \mathbb{Z}$ ). Indeed, note that for any $l \in \mathbb{Z}$ the functor $W_{\leqslant l}$ (see condition (iv') of Theorem प2. $)$ is idempotent and exact; hence the class of objects of $\underline{A}_{\leqslant l}=W_{\leqslant l}(\underline{H t})$ contains all subobjects and factor-objects of its elements (in $\underline{H t}$ ). Thus the long exact sequences coming from applying $\tau_{=j}$ to the $\underline{C}$-extensions given by Lemma $\mathbb{L}$ (5) yield the reduction in question (by induction; here we also use Remark [.2.3.3(3)).

Lastly, by Remark ㄸ.2.3(2) we have $\underline{C}_{w=0}=\bigoplus_{j \in \mathbb{Z}} \operatorname{Obj}_{j}[-j]$; the result follows immediately.

2. By the previous assertion, it suffices to verify the statement for $X \in \underline{C}^{t=j}$. Then the fact is immediate from the statement that 'nice' filtrations of $X[j]$ yield its nice decompositions.

II1. As noted in the proof of Theorem $\square \cdot \mathcal{L}$, this functor is the restriction to $\underline{H t}$ of the functor $b_{i}$ that is right adjoint to the embedding $\underline{C}_{\leqslant i} \rightarrow \underline{C}$. The result follows immediately.

2. Dual to the previous assertion (see Remark ए.2.3(3)).

III All of these assertions are easy consequences of the existence of a weight filtration for $\underline{H t}$ (see Definition $\underline{L}$.

The functors $G r_{i}$ and $G r_{i}^{\prime}$ are exact being the compositions of exact functors. They obviously take their values in $\underline{A}_{i}$ and are identical on it; hence they are idempotent.

Now, we can compute $G r_{i}$ using the following functorial short exact sequence

$$
0 \rightarrow W_{\leqslant i-1} X \rightarrow W_{\leqslant i} X \rightarrow G r_{i} X \rightarrow 0
$$

we also consider its dual

$$
0 \rightarrow G r_{i}^{\prime} X \rightarrow W_{\geqslant i} X \rightarrow W_{\geqslant i+1} X \rightarrow 0 .
$$

We obtain that both of $G r_{i}$ and $G r_{i}^{\prime}$ kill $\underline{C}^{t=0} \cap \underline{C}_{w \geqslant i+1}$ and $\underline{C}^{t=0} \cap \underline{C}_{w \leqslant i-1}$. Since any object of $\underline{H t}$ can be presented as an extension of an object of $\underline{A}_{i}$ by that of $\underline{H t} \cap \underline{C}_{w \geqslant i+1}$ and by an object of $\underline{H t} \cap \underline{C}_{w \leqslant i-1}$, we obtain that $G r_{i} \cong G r_{i}^{\prime}$, whereas (区) yields the last statement in assertion III1.

Next, (四) yields that $G r_{i} X=0$ whenever $W_{\geqslant i+1} X \cong W_{\geqslant i} X$; (四) also yields that this is equivalent to $W_{\leqslant i} X \cong W_{\leqslant i-1} X$. Thus we obtain assertion III2.

Remark 1.2.5. 1. So $\tau_{\leqslant i}$ and $\tau_{\geqslant i}$ preserve $\underline{C}_{w=0}$.

This assumption on $w, t$ is somewhat weaker than their transversality (in contrast to condition (iv) of Theorem ए.2. imply the semi-simplicity of the corresponding $\underline{A}_{i}$. For example, let $\underline{A}$ be a non-semisimple abelian category such that any object of $\underline{A}$ has finite projective dimension; then $\underline{C}=D^{b}(\underline{A}) \cong K^{b}(\operatorname{Proj} \underline{A})$. Then we can consider the 'stupid' weight structure on $C$ whose heart is $\operatorname{Proj} A$. Certainly, $H w$ is preserved by the truncations with respect to the canonical $t$-structure for $\underline{C}$. Yet if we put $\underline{A}_{0}=\operatorname{Proj} \underline{A}$ and all other $\underline{A}_{i}=0$, non-projective objects of $\underline{H t} \cong \underline{A}$ would have no filtrations whose factors are objects of $\underline{A}_{i}$.

One can also consider the direct sum of a collection of ('shifted') examples of this sort in order to get more than one non-zero $\underline{A}_{i}$.

2. For $\underline{A}_{\leqslant l}$ as in the proof of assertion I1, and $\underline{A}_{\geqslant l+1}$ being the categorical kernel of $W_{\leqslant l}(-): \underline{H t} \rightarrow \underline{H t}$ we can re-formulate assertion I2 as follows: $X \in \underline{C}_{w \geqslant 0}$ (resp. $\left.X \in \underline{C}_{w \leqslant 0}\right)$ whenever $X^{\tau=i} \in \underline{A}_{\geqslant i}\left(\operatorname{resp} . X^{\tau=i} \in \underline{A}_{\leqslant i}\right)$ for all $i \in \mathbb{Z}($ for $X \in \operatorname{Obj} \underline{C})$. 
This statement corresponds to the definition of weights for mixed Hodge complexes (by Deligne) and for complexes of mixed Hodge modules (by Saito); see $\S[2.3$ below. For Artin-Tate motives over a number field this result was established in Theorem 3.8 of [Wil08].

We are also able to prove a simple formula for the Grothendieck group of $\underline{C}$.

Proposition 1.2.6. Define $K_{0}(\underline{C})$ as a group whose generators are $[C], C \in \operatorname{Obj} \underline{C}$; if $D \rightarrow B \rightarrow C \rightarrow D[1]$ is a distinguished triangle then we set $[B]=[C]+[D]$.

Let $\underline{C}$ possess transversal $t$ and $w$. Then $K_{0}(\underline{C})$ is a free abelian group with a basis indexed by isomorphism classes of indecomposable objects of all of $\underline{A}_{i}$ (for $i$ running through all integers).

Proof. As we have already noted, $\underline{H w}$ is idempotent complete since all $\underline{A}_{i}[-i]$ are (by Lemma ㅁ..ㅁ. (6)). By Theorem 5.3.1 of [Bon10a] we obtain that $K_{0}(\underline{C}) \cong K_{0}(\underline{H w})$. Recall here that the Grothendieck group of $\underline{H w}$ is defined as the group whose generators are of the form $[X], X \in O b j \underline{H w}$; the relations are $[X]=[Y]+[Z]$ if $X \cong Y \bigoplus Z$ for $X, Y, Z \in O b j \underline{H w}$.

Now, obviously $K_{0}\left(\underline{A}_{i}[-i]\right) \cong K_{0}\left(\underline{A}_{i}\right)$ is a free abelian group with a basis formed by isomorphism classes of indecomposable objects of $\underline{A}_{i}$ (for any fixed $i \in \mathbb{Z}$ ). Besides, we have a functor $\bigoplus \underline{A}_{i}[-i] \rightarrow \underline{H w}$; it induces a surjection of $K_{0}$-groups since it is surjective on objects. This surjection is also injective since it is split by the map $K_{0}\left(\bigoplus_{i \in \mathbb{Z}} \tau_{=i}\right)$.

\section{Examples of transversality}

In this section we construct several examples of transversal weight and $t$-structures. In $\oint \underline{E}$. $\underline{A}$ possesses a weight filtration. In $\oint \mathbb{Z}$ we study 1-motivic examples of this situation (and relate them with the Chow weight structure for Voevodsky's $D M_{g m}$ ), whereas

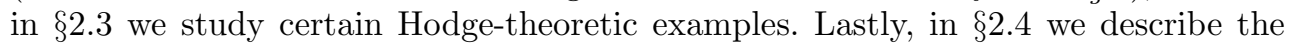
conjectural relations between various 'structures' for $D M_{g m}$.

\subsection{On the main series of examples}

We introduce the notion of a weight filtration for an abelian category following Definition E7.2 of [B-VK10].

Definition 2.1.1. For an abelian $\underline{A}$, we will say that an increasing family of full subcategories $\underline{A}_{\leqslant i} \subset \underline{A}, i \in \mathbb{Z}$, yield a weight filtration for $\underline{A}$ if $\cap_{i \in \mathbb{Z}} \underline{A}_{\leqslant i}=\{0\}, \cup_{i \in \mathbb{Z}} \underline{A}_{\leqslant i}=$ $\underline{A}$, and there exist exact right adjoints $W_{\leqslant i}$ to the embeddings $\underline{A}_{\leqslant i} \rightarrow \underline{A}$.

One can also define weight filtrations via certain exact subfunctors of $\underline{A}$.

Lemma 2.1.2. Let $i$ run through all integers.

1. Let $\underline{A}_{\leqslant i}$ yield a weight filtration for $\underline{A}$. Then they are exact abelian subcategories of $\underline{A}$. Moreover, the corresponding adjunctions yield functorial embeddings $W_{\leqslant i} X \rightarrow X$ for all $X \in O b j \underline{A}$ such that $W_{\leqslant i} X \subset W_{\leqslant i-1} X$ for all $i \in \mathbb{Z}$, and the functors $W_{\geqslant i}: X \mapsto X / W_{\leqslant i-1} X$ are exact also. Besides, the categories $\underline{A}_{i}$ being the 'kernels' of the restriction of $W_{\leqslant i-1}$ to $\underline{A}_{\leqslant i}$, are abelian, and $\underline{A}_{i} \perp \underline{A}_{j}$ for any $j \neq i$. 
2. Let $W_{\leqslant i}$ be a increasing sequence of subfunctors of $1_{\underline{A}}$ such that for any $X \in O b j \underline{A}$ we have: $W_{\leqslant j} X=0$ for $j$ small enough and $=X$ for $j$ large enough. Then taking $\underline{A}_{\leqslant i}$ whose objects are $\left\{X \in O \operatorname{Obj} \underline{A}: W_{\leqslant i}(X) \cong X\right\}$ we obtain a weight filtration corresponding to these $W_{\leqslant i}$. Moreover, all $W_{\leqslant i}$ are idempotent functors.

Proof. Immediate from Proposition E7.4 and Remark E7.8 of [B-VK]0].

Now we prove the statement that will be used for the construction of all the (main) examples of this paper.

Proposition 2.1.3. Suppose that $\underline{A}$ is equipped with a weight filtration, and that all of the corresponding $\underline{A}_{i}$ are semi-simple. Then $\underline{A}_{i}$ yield a strongly semi-orthogonal generating system in $D^{b}(\underline{A})$, and the corresponding weight structure is transversal to the canonical t-structure for $D^{b}(\underline{A})$.

Proof. $\left\{\underline{A}_{i}\right\}$ are obviously generating. The orthogonality statements required are immediate from Remark E7.8, Lemma E7.5, and Proposition E7.4(4) of ibid.

Remark 2.1.4. 1. There is a funny way to make a series of new examples of transversal weight and $t$-structures from one given example.

Suppose that a category $\underline{C}$ with a bounded weight structure $w$ admits a differential graded enhancement (see Definition 6.1.2 of [Bonl0a]; note that one can easily find an enhancement for $D^{b}(\underline{A})$ for any small $\underline{A}$, since a localization of an enhanceable triangulated category is enhanceable). Then for any $N \geqslant 0$ there exist a triangulated category $\underline{C}_{N}$ and an exact truncation functor $t_{N}: \underline{C} \rightarrow \underline{C}_{N}$ such that: $\underline{C}=\left\langle t_{N}\left(\underline{C}_{w=0}\right)\right\rangle ; \underline{C}_{N}\left(t_{N}(A), t_{N}(B)\right)$ for $A \in \underline{C}_{w=i}, B \in \underline{C}_{w=0}(i \in \mathbb{Z})$ is zero for for $i>N$ and $i<0$, and is isomorphic via $t_{N}$ to $\underline{C}(A, B)$ for $0 \leqslant i \leqslant N$ (see Remark 6.2.2 and $\S 6.3$ of [Bon]0a]). In particular, for $N=0$ we obtain the strong weight complex functor $t: \underline{C} \rightarrow \underline{C}_{0} \cong K^{b}(\underline{H w})$; see loc.cit.

We obtain: for a strongly semi-orthogonal generating family of semi-simple $\left\{\underline{A}_{i} \subset \underline{C}\right\}$ (and the corresponding $w$ ) the family $\left\{t_{N}\left(\underline{A}_{i}\right)\right\}$ is also strongly semiorthogonal and generating in $\underline{C}_{N}$ (since for any $i, j, s \in \mathbb{Z}, X \in O b j \underline{A}_{i}, Y \in \operatorname{Obj} \underline{A}_{j}$, the group $\underline{C}_{N}\left(t_{N}(X), t_{N}(Y)[s]\right)$ is either 0 , or is isomorphic to $\left.\underline{C}(X, Y[s])\right)$; moreover, $t_{N}\left(\underline{A}_{i}\right)$ are semi-simple.

So, using any of the examples (of transversal $t$ and $w$ ) described below, one obtains the existence of transversal $w$ and $t$ for all the corresponding $K^{b}(\underline{H w})$ and also for their 'higher' analogues (i.e., the corresponding $\underline{C}_{N}$ for $N>0$ ). In particular, the 'motivic' conjectures imply the existence a $t$-structure transversal to the 'stupid' weight structure (the latter is the 'simplest' weight structure with the heart $=$ Chow, that corresponds to the stupid truncations of complexes; see $\S 1.1$ of [Bon10a]) for $K^{b}$ (Chow); this is true unconditionally for the '1-motivic' part of this category.

The author doubts that $\underline{C}_{N}$ for $\underline{C}=D^{b}(\underline{A})$ (as in the proposition) are always isomorphic to the derived categories of the corresponding $\underline{H t}_{N}$; in any case, this construction surely produces some 'new' examples from the ones that we will describe below.

Note also that twisted complexes over differential graded categories (as defined in [BمK90]; see also $\S 2$ of [Bon09a]) can easily be applied in order to construct (non-trivial) examples of transversal $w$ and $t$ starting from a family of semi-simple $\underline{A}_{i}$. 
2. Below we will consider several motivic and 'Hodge-theoretic' examples of our setting. All the motives, Hodge structures, complexes, and modules, and connecting functors between them that we will consider below will have rational coefficients. This is because the results of this paper cannot be applied (directly) to motives with integral coefficients. Indeed, even the category of finitely generated abelian groups (the 'easiest' part of motives of weight zero) is not semi-simple.

Note also that people usually do not expect Voevodsky's motives with integral coefficients to possess a 'reasonable' motivic $t$-structure (see $\S 3.4$ and Proposition 4.3.8 of $[\nabla \mathbf{0 e} 00]$ ).

\subsection{1-motives; their relation with Voevodsky's motives (endowed with the Chow weight structure)}

First we consider the triangulated category $D^{b}\left(\mathcal{M}_{1}\right)$ of 1-motives.

Proposition 2.2.1. Let $S$ be connected and regular essentially of finite type over $k$. Then the category $D^{b}\left(\mathcal{M}_{1}\right)(S)$ (the derived category of Deligne's 1-motives over $S$; see Appendix C.12 of [B-VKT] $)$ is equipped with a weight structure $w_{1}$ that is transversal to the canonical $t$-structure for it.

Proof. By Proposition C12.1 of ibid., the category $\mathcal{M}_{1}=\underline{A}$ satisfies the conditions of Proposition [.].3.

Next we set $S=$ Spec $k$ and recall that the category $D M_{g m}^{\text {eff }}\left(\subset D M_{g m}\right)$ of effective geometric Voevodsky's motives over $k$ possesses a certain Chow weight structures whose heart is $C h o w^{\text {eff; }}$; see $\S 6.6$ of [Bon10a].

Proposition 2.2.2. 1. The embedding $T: D^{b}\left(\mathcal{M}_{1}\right) \rightarrow D M_{g m}^{\text {eff }}$ defined in $\mathbb{B}-\mathbf{V} \mathbb{1 0}$, Theorem 2.1.2] is weight-exact (i.e., $T\left(D^{b}\left(\mathcal{M}_{1}\right)_{w_{1} \leqslant 0}\right) \subset D M_{g m, w_{\text {Chow }} \leqslant 0}^{\text {eff }}$ and $\left.T\left(D^{b}\left(\mathcal{M}_{1}\right)_{w_{1} \geqslant 0}\right) \subset D M_{g m, w_{\text {Chow }} \geqslant 0}^{\text {eff }}\right)$.

2. The functor LAlb: $D M_{g m}^{e f f} \rightarrow D^{b}\left(\mathcal{M}_{1}\right)$ introduced in Definition 5.2 .1 of ibid. is weight-exact also, as well as RPic (see Definition 5.3 .1 of ibid.; since RPic is contravariant, here weight-exactness means that $\operatorname{RPic}\left(D M_{g m, w_{\text {Chow }} \leqslant 0}^{\text {eff }}\right) \subset D^{b}\left(\mathcal{M}_{1}\right)_{w_{1} \geqslant 0}$, and $\left.\operatorname{RPic}\left(D M_{g m, w_{C h o w} \geqslant 0}^{e f f}\right) \subset D^{b}\left(\mathcal{M}_{1}\right)_{w_{1} \leqslant 0}\right)$.

Proof. 1. Since $w_{1}$ is bounded, it suffices to verify that $T\left(D^{b}\left(\mathcal{M}_{1}\right)_{w_{1}=0}\right) \subset$ ObjChow ${ }^{\text {eff }}$.

To this end it suffices to prove (in the notation of Theorem $\square \cdot 2$ ) that $\underline{A}_{i}[-i] \subset C h o w^{e f f}$. This is immediate from the description of $\underline{A}_{i}$ that can be immediately obtained from Definition C11.1 of [B-VKJ0] along with Lemma 16.1.1 of ibid.

2. It suffices to verify that LAlb and RPic map Chow motives into Chow ones.

Now, (for any $X \in O b j D M_{g m}^{\text {eff }}$ ) $\operatorname{LAlb}(X)$ can be obtained from $L_{i} \operatorname{Alb}(X)[i]$ (see Definition 8.1.1 of ibid.) via extensions (as usual for homology coming from a $t$ structure). Since the heart of a weight structure is always extension-stable, it suffices to verify that $L_{i} \mathrm{Alb}[i]$ sends any smooth projective variety $P / k$ into $C h o w^{e f f}$. We have $L_{i} \operatorname{Alb}(P)=0$ for $i \neq 0,1,2$. Moreover, Corollary 10.2.3 of ibid. immediately implies that $L_{i} \operatorname{Alb}(P)[i]$ is a Chow motif for $i=0,2$. The case $i=1$ is immediate from Lemma 16.1.1 of ibid. 
The result for RPic follows easily, since the functors are interchanged by Poincare duality (see Corollary 5.3.2 of ibid.); note that Poincare duality maps Chow motives into Chow ones. Alternatively, one could apply Corollary 10.6.1 of ibid. (combined with Lemma 16.1 .1 of ibid.).

Remark 2.2.3. 1. Very probably, an analogue of (at least) part 1 of the proposition is also fulfilled for motives over any $S$ that is regular and essentially of finite type over $k$. Recall that a certain version of Voevodsky's $S$-motives (with rational coefficients) was thoroughly studied in [CiDOM]; a Chow weight structure for this category was introduced in [Heb1]] and in [Bon10b]. The main difficulty here is to construct a comparison functor.

2. As shown in $\S 2$ of [BonT0a], for any (co)homology theory defined on $\underline{C}$ a weight structure $w$ for it yields certain weight filtrations (cf. Definition 1 below), weight spectral sequences (cf. Proposition [.工. (I)), and virtualt-truncations. The Proposition immediately yields the compatibility of all of these notions for $D^{b}\left(\mathcal{M}_{1}\right)$ and $D M_{g m}^{e f f}$ (with respect to $T$, LAlb, and RPic). Also, these comparison functors respect the weight complex functor (see $\S 3$ of ibid. and $\S 6.3$ of [Bon09a]).

3. An analogue of Proposition [2.2. (along with Proposition ए.2.2(1)) for ArtinTate motives over a number field was established in $\S 3$ of [Wil08].

\subsection{Graded polarizable mixed Hodge complexes and Hodge modules; the Hodge realization}

Proposition 2.3.1. I Let $X$ be a complex variety.

1. There exists a weight structure $w$ on the category $D^{b} M H M(X)$ (the derived category of mixed Hodge modules over $X$; see [Sai8.]]) that is transversal to the canonical $t$-structure for it.

2. For this weight structure $D^{b} M H M(X)_{w \leqslant 0}$ is the class of complexes of mixed Hodge modules of weight $\leqslant 0$, and $D^{b} M H M(X)_{w \geqslant 0}$ is the class of complexes of mixed Hodge modules of weight $\geqslant 0$ in the sense of Definition 1.6 of ibid.

II Let $k$ be a subfield of the field of complex numbers.

1. There exists a weight structure $w_{\text {Hodge }}$ for the category $D_{\tilde{H}_{p}}^{b}(k)$ introduced in $\S 3$ of [Bei86]], that is transversal to the canonical t-structure for it (given by Lemma 3.11 of ibid.).

2. The (contravariant) Hodge realization functor $R^{\operatorname{sing}}(k): D M_{g m}(k) \rightarrow D_{\tilde{H}_{p}}^{b}(k)$ (for example, the composition of the 'polarizable mixed realization' one constructed in §2.3 of [Hub00] with the natural functor of 'forgetting all other realizations'; cf. $\$ 17.2$ of [B-VK $[0]$ ) is weight-exact with respect to these weight structures, i.e.,

$$
R^{\text {sing }}\left(D M_{g m, w_{\text {Chow }} \leqslant 0}(k)\right) \subset D_{\tilde{H}_{p}}^{b}(k)_{w_{\text {Hodge }} \geqslant 0}
$$

and

$$
R^{\text {sing }}\left(D M_{g m, w_{\text {Chow }} \leqslant 0}(k)\right) \subset D_{\tilde{H}_{p}}^{b}(k)_{w_{H o d g e} \leqslant 0} .
$$

Proof. I1. We verify that $M H M(X)$ satisfies the conditions of Proposition $2 \perp$. This is immediate from Propositions 1.5 and 1.9 of [Sai89].

2. Immediate from Definition 1.6 of [Sai89] along with Remark ए2. (2). 
II1. By Lemma 3.11 of [Bei86], $D_{\tilde{H}_{p}}^{b}(k)$ is isomorphic to the bounded derived category of the abelian category of graded polarizable mixed Hodge structures (over $k$ ). Similarly to the proof of assertion I1, it remains to apply Proposition [2].3.

2. As in the proof of Proposition L2.2, we should verify that $R^{\text {sing }}$ maps the heart of $w_{\text {Chow }}(k)$ into that of $w_{\text {Hodge }}(k)$. To this end it suffices to note that the $i$-th cohomology of a smooth projective $P / \mathbb{C}$ is a pure (polarizable) Hodge structure of weight $i$ (for $i \in \mathbb{Z})$.

Remark 2.3.2. 1. The weight filtration on $D_{\tilde{H}_{p}}^{b}$ obtained corresponds to the Deligne's definition of weights for mixed Hodge complexes (cf. assertion I2).

2. For a certain category of Beilinson motives over $X$ (these are relative Voevodsky's motives with rational coefficients over $X$; see [CiDn:] ) a certain Chow weight structure was introduced in [Heb1]] and [Bon106] $)$. Very probably, this weight structure is compatible (similarly to assertion II2) with the one introduced in assertion I1 (and so with Saito's weights for complexes of mixed Hodge modules); note also that the 'functoriality' properties of the weights of the latter (see Proposition 1.7 of [Sai89]) are parallel to those for $X$-motives (as described in Theorem 3.7

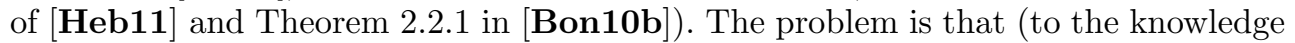
of the author) no 'mixed Hodge module' realization of $X$-motives is known to exist at the moment.

3. It does not seem very difficult to extend assertion II to Huber's category $D_{\mathcal{M R}^{\mathcal{P}}}$ of mixed realizations (introduced in $\S 21$ of [Hub95]]), the corresponding cohomology

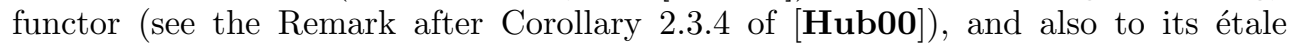
cohomology analogue.

On the other hand, for a finite characteristic $k$ one cannot prove that the étale cohomology of smooth projective varieties is polarizable in absence of the Hodge standard conjecture (for this case). Hence one can only define realizations with values in categories that do not satisfy any polarizability conditions; whereas without these restrictions there will exist non-trivial 1-extensions inside $\underline{A}_{i}$ for a single $i$ (and so, $\underline{A}_{i}[-i]$ will not be contained in the heart of any weight structure).

\subsection{Mixed motives: the conjectural picture}

It is widely believed that Voevodsky's $D M_{g m}$ (over a base scheme $S$ ) possesses the so-called motivic $t$-structure, whose heart $M M(S)$ (the category of mixed motivic

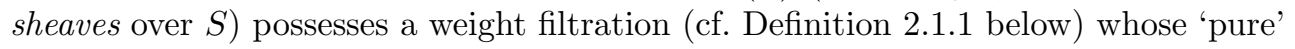
factors $\underline{A}_{i}$ are abelian semi-simple (see $\S 5.10 \mathrm{~A}$ in [Bei87]). Moreover, at least in the case when $S$ is the spectrum of a (perfect) field $k$, people believe that all of $\underline{A}_{i}[-i]$ consist of Chow motives. These conjectures yield (immediately by Theorem प्.2. that the Chow weight structure for $D M_{g m}(S)$ (that is known to exist unconditionally) is transversal to the motivic $t$-structure. Thus the results of Section 1.2 can be applied to this situation. We study these questions in detail and obtain some new (conjectural) information on motives in [Bon 110$]$ this way. Note that 'classically' people were looking only for the mixed motivic $t$-structure and for the 'weight filtration' for $D M_{g m}$; cf. the introduction and Remark 2.4. below. The notion of weight structure is new in this picture; it allows us to construct certain 'weights' for motives unconditionally. 
Now we show that the notion of transversal $t$ - and weight structures indeed yields a way to relate the 'classical' approach to weights with 'our' (i.e., the 'Chow weight structure') one. For $X \in D M_{g m}^{t=0}$, a nice decomposition of $X$ (see condition (iv) of Theorem प.2. of the Theorem). Now, for any (contravariant) cohomology theory $H: D M_{g m} \rightarrow \underline{A}$ the $-1-j$-th level of the 'weight filtration' of $H^{i}(X)=H(X[-i])\left(X \in O b j D M_{g m}\right.$, $i, j \in \mathbb{Z}$ ) is 'classically' defined as $H^{i}\left(X_{2}\right)$, where $X_{2}$ is taken from (四). Now suppose that $H$ factorizes through $M M$ (i.e., it is the composition of the functor $M \mapsto M^{\tau_{M M}=0}$ with a contravariant exact functor; note that conjecturally one can factorize through $M M$ all cohomology theories endowed with 'classical' weights); then $H^{i}(X)$ and its weight filtration coincides with those for $X^{\prime}=\tau_{=-i} X$ (here we use the fact that the weight filtration functors given by Lemma ㄷ. . $($ (II) commute with $t$-truncations). Hence

$$
\begin{aligned}
H^{i}\left(X_{2}\right) \cong H^{i}\left(X_{2}^{\prime}\right) \cong \operatorname{Im}\left(H^{i}\left(w_{\geqslant j+1-i} X^{\prime}\right) \rightarrow H^{i}\left(X^{\prime}\right)\right) & \\
& \cong \operatorname{Im}\left(H^{i}\left(w_{\geqslant j+1-i} X\right) \rightarrow H^{i}(X)\right) .
\end{aligned}
$$

Now note that the last two terms do not depend on the choices of the corresponding weight decompositions (see Proposition 2.1.2 of [Bon10a] or Definition [.] below). Hence one can define the weight filtration of $H^{j}(X)$ unconditionally (using the last term of the formula)!

One can also make a similar observation using a 'weight' filtration on $\underline{A}$ (if it exists); see Remark 2.4.3 of [Bon10a].

Whereas the approach for defining weights for cohomology described (above) is somewhat 'cheating' when we apply it to pure motives (since it usually gives no new information); yet it yields interesting results in the 'mixed' case. Note here: for $X=\mathcal{M}(P)$, where $P$ is smooth projective over $k$ (or over the corresponding base $S$ ), the motives $X_{1}, X_{2}$ should come from a Chow-Kunneth decomposition of $X$; so their construction is completely out of reach at the moment (in general).

Remark 2.4.1. One can take the setting of Lemma ㄷ.1.5(II3) for the definition of a weight filtration for a triangulated category; see also the equivalent Definition E17.1 of [B-VK10] (this is a 'triangulated analogue' of Definition $[\mathbf{D}$ ).

Now we describe how one could obtain a weight filtration for $D M_{g m}$ assuming that the motivic $t$-structure exists. If one has transversal $t, w$ for $\underline{C}$, then using the corresponding $\underline{A}_{i}$ one can define the triangulated categories $\underline{C}_{i}=\left\langle\underline{A}_{i}\right\rangle$. Next one can use Lemma $\square . \mathcal{L}$ (I) and introduce the corresponding categories $\underline{C_{\leqslant}}$.

It is easily seen that in the case we consider in this paragraph (i.e., for $\underline{C}=D M_{g m}$, the corresponding 'structures' and $\underline{A}_{i}$ ) we should obtain the long-searched-for weight filtration for $D M_{g m}$ (that was already mentioned in the introduction).

\section{On weight spectral sequences and weight filtrations for $H t$}

In this section we study the relations between $w, t$, weight filtrations, and weight spectral sequences. In particular, we study a condition on $w$ and $t$ that is strictly weaker than their transversality (yet it is somewhat easier to verify). 
In $\S$. in [Bon10a] ); and relate the degeneration of the latter with 'exactness' of the corresponding filtrations for homology. In $\$$.2 we study the case when a homology theory comes from a $t$-structure; we obtain a certain weight filtration for $\underline{H t}$ in this case.

\subsection{On weight filtrations and (degenerating) weight spectral sequences for homology}

First we recall weight filtrations and weight spectral sequences (as introduced in [Bon]0a]).

Let $\underline{A}$ be an abelian category. In $\S 2$ of ibid. for $H: \underline{C} \rightarrow \underline{A}$ that is either cohomological or homological (i.e., it is either covariant or contravariant, and converts distinguished triangles into long exact sequences), certain weight filtrations and weight spectral sequences (corresponding to $w$ ) were introduced. Below we will consider the homological functor case; certainly, one can pass to cohomology (that often seems to be somewhat more common) by a simple reversion of arrows (cf. $\S 2.4$ of ibid.).

Definition 3.1.1. Let $H: \underline{C} \rightarrow \underline{A}$ be a homological functor, $i \in \mathbb{Z}$.

1. We denote $H \circ[i]: \underline{C} \rightarrow \underline{A}$ by $H_{i}$.

2. We choose some $w_{\leqslant i} X$ and define the weight filtration for $H$ by $W_{i} H: X \mapsto$ $\operatorname{Im}\left(H\left(w_{\leqslant i} X\right) \rightarrow H(X)\right)$.

Recall that $W_{i} H$ is functorial in $X$ (in particular, it does not depend on the choice of $\left.w_{\leqslant i} X\right)$; see Proposition 2.1.2(1) of ibid. (this fact also easily follows from

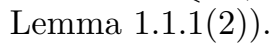

Now we recall some of the properties of weight spectral sequences, and prove some easy (new) results in the case when they degenerate.

Proposition 3.1.2. Let $H: \underline{C} \rightarrow \underline{A}$ be a homological functor.

$I$ For any $X \in O b j \underline{C}$ there exists a spectral sequence $T=T_{w}(H, X)$ with $E_{1}^{p q}(T)=$ $H_{q}\left(X^{p}\right)$ for certain $X^{i} \in \underline{C}_{w=0}$ (coming from certain weight decompositions as in

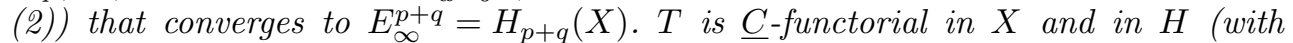
respect to composition of $H$ with exact functors of abelian categories) starting from $E_{2}$. Besides, the step of filtration given by $\left(E_{\infty}^{l, m-l}: l \geqslant k\right)$ on $H_{m}(X)$ (for some $k, m \in \mathbb{Z})$ equals $\left(W_{-k} H_{m}\right)(X)$. Moreover, $T(H, X)$ comes from an exact couple with $D_{1}^{p q}=H_{p+q}\left(w_{\leqslant-p} X\right)$ (here one can fix any choice of $\left.w_{\leqslant-p} X\right)$.

We will say that $T$ degenerates at $E_{2}$ (for a fixed $H$ ) if $T_{w}(H, X)$ does so for any $X \in O b j \underline{C}$.

II Suppose that $T$ degenerates at $E_{2}$ (as above), $i \in \mathbb{Z}$. Then the following statements are fulfilled.

1. The functors $W_{i} H$ and $W_{i}^{\prime} H: X \mapsto H(X) / W_{i} H(X)$ are homological.

2. For any $f \in \underline{C}(X, Y)$ the morphism $H(f)$ is strictly compatible with the filtration of $H$ by $W_{i}$ i.e., $W_{i} H(X)$ surjects onto $W_{i} H(Y) \cap \operatorname{Im} H(f)$.

3. Let $\underline{B}$ be an abelian category; $F: \underline{A} \rightarrow \underline{B}$ be an exact functor. Then $T(F \circ H,-)$ degenerates also.

III Conversely, if $F$ (as in assertion II3) is conservative and $T(F \circ H,-)$ degenerates, then $T$ degenerates also.

Proof. I All of the results stated were verified in Theorem 2.3.2 of ibid. (see formula (13) for a precise description of the corresponding filtration), expect the fact that the 
functoriality of $T$ with respect to $H$ does not depend on the choice of the corresponding weight decompositions. The latter assertion is immediate from Theorem 2.4.2(II) of [Bonloc].

II1. The degeneration at $E_{2}$ yields that $\left(W_{-p} H_{p+q}\right) X \cong D_{2}^{p q} T(H, X)$ for any $X \in O b j \underline{C}$ (so, here we consider the derived exact couple). Now, we note that the right hand side of this isomorphism is homological in $X$ (this is the corresponding virtual $t$-truncation of $H$; see Proposition 2.5(II1) of [Bon10a]). We deduce that $W_{i}^{\prime} H$ is homological also by applying part II2 of loc.cit.

2. We complete $f$ to a distinguished triangle $X \stackrel{f}{\rightarrow} Y \stackrel{g}{\rightarrow} Z$. Then

$$
W_{i} H(Y) \cap \operatorname{Im} H(f)=W_{i} H(Y) \cap \operatorname{Ker} H(g)=\operatorname{Ker}\left(W_{i} H(Y) \rightarrow W_{i} H(Z)\right) .
$$

It remains to note that the last term coincides with $\operatorname{Im} W_{i} H(X)$, since $W_{i} H$ is homological.

3. Obvious.

III Easy; note that conservative exact functors of abelian categories do not kill non-zero morphisms.

\subsection{On the weight filtration for the heart of $t$}

We fix certain (bounded) $w$ and $t$ for $\underline{C}$, and consider certain conditions ensuring that $w$ induces a weight filtration for $\underline{A}=\underline{H t}$ (in the sense of Definition $\underline{\Sigma} \boldsymbol{L}$ ).

Proposition 3.2.1. Let $H=(-)^{\tau=0}$; let $i$ run through all integral numbers.

$I$ Suppose that the corresponding $T$ degenerates. Then the following statements are fulfilled.

1. The functors $W_{i} H: \underline{C} \rightarrow \underline{H t}$ are homological. The restrictions $W_{\leqslant i}$ of $W_{i} H$ to $\underline{H t}$ define a weight filtration for this category (via Lemma [.T.(2)). Besides, $W_{i} H \cong$ $W_{\leqslant i} \circ H$.

2. For $X \in \underline{C}^{t=0}$ we have: $X \in O \operatorname{Obj} \underline{A}_{i}$ whenever there exists a bounded complex $C=\cdots \rightarrow C^{-1} \rightarrow C^{0} \rightarrow C^{1} \rightarrow \ldots$ in $\underline{H w}$ such that (for $l, j \in \mathbb{Z}$ ) the l-th homology of the $\underline{H \text { t-complex }} C^{\tau=j}$ is isomorphic to $X$ for $l=0, j=i$, and is zero otherwise.

3. For $X \in \underline{C}^{t=0}$, we have: $X \in O b j \underline{A}_{\leqslant i}$ whenever there exist a $Y \in \underline{C}_{w \leqslant i}$ and an epimorphism $H(Y) \rightarrow X$.

II Suppose that $t$ is transversal to $w$. Then $T$ degenerates. The corresponding $\underline{A}_{i}$, $\underline{A}_{\leqslant i}, W_{\geqslant i}$, and $W_{\leqslant i}$ are the same as the ones described in $\S$ प..

III Let $\underline{B}$ be an abelian category; let $F: \underline{H t} \rightarrow \underline{B}$ be an exact functor.

1. Suppose that $T$ degenerates. Then $T_{w}(F \circ H,-)$ also does.

2. Conversely, suppose that $F$ is conservative and $T_{w}(F \circ H,-)$ degenerates. Then $T$ degenerates.

Moreover, for $X \in \underline{C}^{t=0}$ we have: $W_{\leqslant i} X=X \quad$ (resp. $\left.W_{\leqslant i} X=0\right)$ whenever $W_{i}(F \circ H)(X)=F(X)$ (resp. $\left.W_{i}(F \circ H)(X)=0\right)$.

Proof. I1. Immediate from Proposition

2. If $X \in O b j \underline{A}_{i}$, then (by the definition of $\underline{A}_{i}$ ) for the corresponding weight spectral sequences we obtain: $W_{\leqslant i} X=X, W_{\leqslant i-1} X=0$. This translates into (see the proof of Proposition [.]2(II1)): $D_{2}^{-i, i} X \cong X$ and $D_{2}^{1-i, i-1} X=0$. Hence $X \cong E_{2}^{-i, i}$, whereas all the remaining $E_{2}^{p q}$ are zero (note that $E_{2}^{p+q}(T)=0$ for any $X \in \underline{C}^{t=0}, p+q \neq 0$ ). Therefore, $X$ is the $-i$-th homology of the complex $\left(C^{j}\right)=\left(E_{1}^{j i}\right)=\left(H_{i}\left(X^{j}\right)\right)$, whereas 
all the other homology of this complex is zero, as well as the all of the homology of the complexes $\left(X^{j}\right)^{\tau=l}$ for all (fixed) $l \neq 0$.

Conversely, for any $C^{0} \in \underline{C}_{w=0}$ we obviously have $\tau^{=i} C^{0} \in \operatorname{Obj} \underline{A}_{i}$; hence this is also true for any subfactor of $H\left(C^{0}[i]\right)$. Here we only use the fact that $\underline{A}_{i}$ is an exact subcategory of $\underline{A}$; no other restrictions on the corresponding complex $C$ are necessary.

3. By definition, $X \in \underline{A}_{\leqslant i}$ whenever $W_{i} H(X)=X$. Hence for $X \in \underline{A}_{\leqslant i}$ we can take $Y=w_{\leqslant i} X$.

Conversely, let there exist an epimorphism $H(Y) \rightarrow X$ for $Y \in \underline{C}_{w \leqslant i}$. Since $\underline{A}_{\leqslant i}$ is an exact subcategory of $\underline{A}$, we may assume that $X=H(Y)$. Hence $W_{i} H(Y)=H(Y)$. Now, assertion I1 yields that $W_{i} H(Y)=W_{\leqslant i}(H(Y))$; the result follows immediately.

II The previous assertion yields that $E_{1}^{p q} T(H, X) \in \underline{A}_{q}$ for any $p, q \in \mathbb{Z}$ and $X \in$ $\operatorname{Obj} \underline{C}$; hence the same is true for $E_{r}^{p q}$ for any $r \geqslant 1$. Hence the boundary morphisms of $E_{i}(T)$ for $i \geqslant 2$ vanish, since their sources and targets necessarily belong to distinct $\underline{A}_{i}$, and we obtain that $T$ degenerates at $E_{2}$ indeed.

Now, assertions I 2 and I3 easily yield that $\underline{A}_{i}$ and $\underline{A}_{\leqslant i}$ for the corresponding weight filtration of $\underline{H t}$ are the same as the ones described in $\S \square$ (for transversal $w$ and $t$ ). Hence the two versions of $W_{\leqslant i}$ and $W_{\geqslant i}$ coincide also.

III Assertion 1 is just a partial case of Proposition [.2. (II3). Besides, the first part of assertion 2 is also a partial case of part III of ibid.

In order to verify the second part of assertion III2 it suffices to note that exact functors respect weight filtrations for homology, whereas conservative exact functors cannot kill non-zero levels of these filtrations and non-zero $\underline{H t}$-morphisms.

Remark 3.2.2. 1. $T$ also degenerates for the example described in Remark ए2. (1) (i.e., for $\underline{C}=D^{b}(\underline{A}) \cong K^{b}(\operatorname{Proj} \underline{A})$ ). Hence, the degeneration of $T_{w}\left((-)^{\tau=0},-\right)$ is strictly weaker than the transversality of $t$ with $w$.

Unfortunately, the author does not know of any 'description' of $w$ and $t$ in terms of 'generators' (as in Remark प.2.3(2)) in this more general situation.

It would also be interesting to understand whether the degeneration of $T$ implies that $\tau_{=i}$ preserves $\underline{H w}$ (as in the example mentioned), and whether the converse implication is valid.

2. The author does not have a lot of examples of this situation (with $w$ not transversal to $t$ ). The advantage of this weaker condition is that it could be checked 'at $t$-exact conservative realizations' of $\underline{C}$. In particular, conjecturally it is sufficient to verify the degeneration of the (Chow)-weight spectral sequences for the ('perverse') étale (co)homology of (Voevodsky's) motives (instead of the 'mixed motivic homology' that corresponds to the conjectural motivic $t$-structure); cf. $\S 3.3$ of [Bon]16].

\section{Acknowledgements}

The work is supported by RFBR (grants no. 10-01-00287a and 11-01-00588a), by the Saint-Petersburg State University research grant no. 6.38.75.2011, and by the Federal Targeted Programme "Scientific and Scientific-Pedagogical Personnel of the Innovative Russia in 2009-2013" (Contract No. 2010-1.1-111-128-033). 
MIKHAIL V. BONDARKO

\section{References}

[B-VK10] Barbieri-Viale, L.; Kahn, B. On the derived category of 1-motives, preprint, http://arxiv.org/abs/1009.1900.

[BBD82] Beilinson, A. A.; Bernstein, J.; Deligne, P. Faisceaux pervers. Analysis and topology on singular spaces, I (Luminy, 1981), 5-171, Astérisque, 100, Soc. Math. Paris, 1982.

[Bei86] Beilinson, A. A. Notes on absolute Hodge cohomology. Applications of algebraic $K$-theory to algebraic geometry and number theory, Part I, II (Boulder, Colo., 1983), 35-68, Contemp. Math., 55, Amer. Math. Soc., Providence, RI, 1986.

[Bei87] Beilinson, A. A. Height pairing between algebraic cycles. K-theory, arithmetic and geometry (Moscow, 1984-1986), 1-25, Lecture Notes in Math., 1289, Springer, Berlin, 1987.

[BoK90] Bondal, A. I.; Kapranov, M. M. Framed triangulated categories. (Russian) Mat. Sb. 181 (1990), no. 5, 669-683; translation in Math. USSR-Sb. 70 (1991), no. 1, 93-107.

[Bon09a] Bondarko, M. V. Differential graded motives: weight complex, weight filtrations and spectral sequences for realizations; Voevodsky versus Hanamura. J. Inst. Math. Jussieu 8 (2009), no. 1, 39-97, see also http://arxiv.org/abs/ math.AG/0601713.

[Bon09s] Bondarko, M. V. Weight structures and motives; comotives, coniveau and Chow-weight spectral sequences, and mixed complexes of sheaves: a survey, preprint, http://arxiv.org/abs/0903.0091.

[Bon10a] Bondarko, M. V. Weight structures vs. $t$-structures; weight filtrations, spectral sequences, and complexes (for motives and in general). J. K-Theory 6 (2010), no. 3, 387-504, see also http://arxiv.org/abs/0704.4003.

[Bon10b] Bondarko, M. V. Weights for relative motives; relation with mixed complexes of sheaves, preprint, http://arxiv.org/abs/1007.4543.

[Bon10c] Bondarko, M. V. Motivically functorial coniveau spectral sequences; direct summands of cohomology of function fields. Doc. Math. 2010, Extra volume: Andrei A. Suslin sixtieth birthday, 33-117.

[Bon11a] Bondarko, M. V. $\mathbb{Z}[1 / p]$-motivic resolution of singularities. Compos. Math. 147 (2011), no. 5, 1434-1446.

[Bon11b] Bondarko, M. V. Mixed motivic sheaves (and weights for them) exist if 'ordinary' mixed motives do, preprint, http://arxiv.org/abs/1105.0420.

[CiD09] Cisinski, D.-C.; Déglise, F. Triangulated categories of mixed motives, preprint, http://arxiv.org/abs/0912.2110.

[Heb11] Hébert, D. Structure de poids à la Bondarko sur les motifs de Beilinson. Compos. Math. 147 (2011), no. 5, 1447-1462.

[Hub95] Huber, A. Mixed motives and their realization in derived categories. Lecture Notes in Mathematics, 1604. Springer-Verlag, Berlin, 1995. xvi+207 pp. ISBN: 3-540-59475-2. 
[Hub00] Huber, A. Realization of Voevodsky's motives. J. Algebraic Geom. 9 (2000), no. 4, 755-799.

[Pau08] Pauksztello, D. Compact corigid objects in triangulated categories and co-t-structures. Cent. Eur. J. Math. 6 (2008), no. 1, 25-42.

[Sai89] Saito, M. Introduction to mixed Hodge modules. Actes du Colloque de Théorie de Hodge (Luminy, 1987). Astérisque No. 179-180 (1989), 10, 145-162.

[Voe00] Voevodsky, V. Triangulated categories of motives over a field. Cycles, transfers, and motivic homology theories, 188-238, Ann. of Math. Stud., 143, Princeton Univ. Press, Princeton, NJ, 2000.

[Wil08] Wildeshaus, J. Notes on Artin-Tate motives, preprint, http://www.math.uiuc.edu/K-theory/0918/.

Mikhail V. Bondarko mbondarko@gmail.com

St. Petersburg State University, Department of Mathematics and Mechanics, Bibliotechnaya Pl. 2, 198904, St. Petersburg, Russia 